\title{
Usefulness of glycosylated haemoglobin (HbA1c) to screen for diabetes in patients with schizophrenia
}

\author{
Linda Hanssens ${ }^{1}$, Marc De Hert², Dominique Van Eyck², Martien Wampers ${ }^{2}$, Joseph Peuskens ${ }^{2}$, Andre Scheen ${ }^{3}$ \\ ${ }^{1}$ Department of Epidemiology and Public Health, University Liège, Belgium \\ ${ }^{2}$ University Center St Jozef, Catholic University Louvain, Leuvense Steenweg 517, 3070 Kortenberg, Belgium \\ ${ }^{3}$ Department of Diabetology, CHU de Sart Tilman, University Liège, Belgium
}

\section{Dear Editor,}

Metabolic abnormalities have consistently been identified as a part of schizophrenic illness. The interest in this topic has recently been renewed since the introduction of second generation antipsychotics (SGA) and their possible association with metabolic abnormalities (ADA, 2004; De Nayer et al., 2005; Newcomer, 2005; Scheen and De Hert, 2005).

Generally, assessment of fasting glucose is recommended as screening and monitoring for diabetes (ADA/APA consensus, 2004) although an Oral Glucose Tolerance Test (OGTT) remains the gold standard (Expert Committee on the Diagnosis and Classification of Diabetes Mellitus, 2003). HbAlc is accepted as a useful index of mean blood glucose levels in the treatment of patients with diabetes but was not selected by the expert committee as a means for screening (Expert committee on the Diagnosis and Classification of Diabetes Mellitus, 2003; Kilpatrick et al., 1998; Mulkerin et al., 1992; Rohlfmg et al., 2000).

Since $\mathrm{HbA}$ lc testing can be performed at any time of day without special patient preparation it could be a more convenient screening method for both patients and caregivers. A recent consensus on the management of diabetes risk in schizophrenia of British psychiatrists suggests that assessment of HbAlc can be used for screening (Expert Group, 2004).

We evaluated whether assessment of HbAlc is useful to screen for the presence of glucose abnormalities or diabetes in patients with schizophrenia in a large dataset of our prospective metabolic study. The study was approved by an ethical committee and all patients gave written informed consent.

All consecutive patients with a DSM-IV diagnosis of schizophrenia or schizoaffective disorders $(19.9 \%)(N=$ $388)$, both out- (31.7\%) or inpatients, were asked to participate in an extensive screening and prospective followup study of metabolic parameters. For all 378 (excluding patients being treated for diabetes, $77=10$ ), an OGTT, with assessment of HbA1c on the same day (HPLC Menarini HA-8160), was performed at baseline.

All patients were treated with the same antipsychotic medication for at least 6 months. The majority of patients were treated with SGA (88.9\%).

For the diagnosis of diabetes and pre diabetic abnormalities we used the criteria of The American Diabetes Association (Impaired fasting glucose (IFG), fasting glucose $\geq 100 \mathrm{mg} / \mathrm{dl}$; Impaired Glucose Tolerance (IGT), glucose $\geq 140 \mathrm{mg} / \mathrm{dl}$ at $2 \mathrm{~h}$ in the OGTT; diabetes, fasting glucose $\geq 126 \mathrm{mg} / \mathrm{dl}$ or glucose $\geq 200 \mathrm{mg} / \mathrm{dl}$ at $2 \mathrm{~h}$ in the OGTT) (Expert Committee, 1997, 2003).

Based on the results of the OGTT three patient groups were identified: a) patients meeting criteria for diabetes (10 known and treated diabetes, 22 newly detected cases); b) patients with either IFG, IGT or both; c) patients without glucose abnormalities. In all patients with newly detected diabetes and glucose abnormalities the assessment of HbA1c was on the same day as the OGTT.

According to ADA criteria $8.3 \%$ (32) of patients met criteria for diabetes, another $24.2 \%$ (95) met criteria for pre-diabetes (17.8\% IFG, $12.6 \%$ IGT, either isolated or in combination).

HbA1c differs significantly between groups $(p<.0001$, all pair wise comparisons $p<.01)$ (Table 1$)$. Within each patient group large inter-individual differences in HbA1c values were however observed. The HbA1c values also differ significantly between patients being treated for diabetes $(8.5 \pm 2.0 \%)$ and patients with newly detected diabetes $(6.8 \pm 1.4 \%)(p<.01)$. With a cut-off value of $6.3 \%$ (normal threshold reference) the specificity of predicting diabetes (91.9), recent onset diabetes (91.9) or glucose abnormalities (95.5) was high (Table 1). But the sensitivity is only moderate for the prediction of diabetes $(68.8$, missing 1 out of 3 cases, all cases missed are new onset cases), poor for the prediction of new onset diabetes (54.5, nearly missing 1 out 2 cases) or glucose abnormalities (IFG/IGT) (18.9\%). 
Relying only on measurements of HbAlc provides limited predictive value to detect glucose abnormalities and or recent onset diabetes. Screening for glucose abnormalities should be at least by assessment of fasting glucose.

Our results confirm results obtained in normal populations showing that HbA1c cannot be used to screen for or detect recent onset diabetes in schizophrenic patients (Kilpatrick et al., 1998; Mulkerin et al., 1992; Rohlfing et al., 2000). Measuring of HbA1c is only recommended for the follow-up of treatment of patients suffering from diabetes (Expert Committee on the Diagnosis and Classification of Diabetes Mellitus, 2003).

Table 1 HbA1c values in function of glucose abnormalities

\begin{tabular}{lllll}
\hline Patient group & HbA1c (\%) & & \\
\cline { 2 - 5 } & Mean & STD & Min & Max \\
\hline Diabetes & 7.3 & 1.8 & 4 & 12 \\
IFG/IGT/both & 5.6 & 0.7 & 3.8 & 8.8 \\
Normal & 5.3 & 0.5 & 3.4 & 7.3 \\
\hline
\end{tabular}

\section{References}

American Diabetes Association, 2004. Consensus development conference on antipsychotic drugs and obesity and diabetes. Diabetes Care 27, 596-601.

De Nayer, A, De Hert, M., Scheen, A., et al., 2005. Conference report: Belgian consensus on metabolic problems associated with secondgeneration antipsychotics. Int. J. Psychiatry Clin. Pract. 9 (2), 130-137.

Expert Committee on the Diagnosis and Classification of Diabetes Mellitus, 1997. Report of the expert committee on the diagnosis and classification of diabetes mellitus. Diabetes Care 20, 1183-1197.

Expert Committee on the Diagnosis and Classification of Diabetes Mellitus, 2003. Follow-up report on the diagnosis of diabetes mellitus. Diabetes Care 26, 3160-3166.

Expert Group, 2004. 'Schizophrenia and diabetes 2003' expert consensus meeting, Dublin, 3-4 October: consensus summary. Br. $J$. Psychiatry 47 Suppl., S112-S114.

Kilpatrick, E.S., Maylor, P.W., Keevil, B.G., 1998. Biological variations of glycated hemoglobin: implications for diabetes screening and monitoring. Diabetes Care 21, 261-264.

Mulkerin, E.C., Arnold, J.D., Dewar, et al., 1992. Glycosylated haemoglobin in the diagnosis of diabetes mellitus in elderly people. Age Ageing 21, 175-177.

Newcomer, J.W., 2005. Second-generation (atypical) antipsychotics and metabolic effects: a comprehensive literature review. CNS Drugs 19 (Suppl. 1), 1-93.

Rohlfmg, C, Little, R., Wiedmeyer, H., et al., 2000. Use of $\mathrm{GHb}\left(\mathrm{HbA}_{1 \mathrm{c}}\right)$ in screening for undiagnosed diabetes in the US population. Diabetes Care 23, 187-191.

Scheen, A.J., De Hert, M., 2005. Drug-induced diabetes mellitus: the example of atypical antipsychotics. Rev. Med. Liege 60 (5-6), 455-460. 